Journal of Applied Fluid Mechanics, Vol. 9, Special Issue 1, pp. 121-129, 2016. Selected papers from the $7^{\text {th }}$ International Exergy, Energy

and Environment Symposium, IEEE7-2015

Available online at www.jafmonline.net, ISSN 1735-3572, EISSN 1735-3645.

DOI: $10.36884 / \mathrm{jafm} . \overline{9}$.SI1.25823

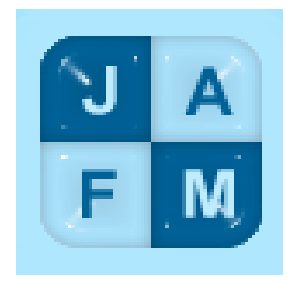

\title{
Experimental and Numerical Investigations of the Characterisation of Reflected Overpressures around a Complex Structure
}

\author{
L. Blanc ${ }^{1,2}$, J-L. Hanus ${ }^{1 \dagger}$, M. William-Louis ${ }^{3}$ and B. Le-Roux ${ }^{2}$ \\ ${ }^{1}$ INSA-CVL, Univ. Orléans, PRISME, EA 4229,88 boulevard Lahitolle, Bourges, 18022 cedex, France \\ ${ }^{2}$ INERIS , Parc Technologique ALATA, BP2 60550, Verneuil-en-Halatte, France \\ ${ }^{3}$ Univ. Orléans, IUT, PRISME, EA 4229,63 Avenue du Maréchal de Lattre de Tassigny, 18000, Bourges,
}

France

†CorrespondingAuthor Email: jean-luc.hanus@insa-cvl.fr

(Received October 20, 2015; accepted December 10, 2015)

\begin{abstract}
In explosion-structure interaction problems, an accurate prediction of blast loading remains a hard challenge. The reflected overpressures around a complex structure, such as a building with an apse and an atrium are almost always unpredictable so that experiments and numerical simulations may be the only possibilities to evaluate the threat of an industrial explosion. Well instrumented blast experimental studies are first carried out at small scale on a rigid specimen with a variable incidence angle. The main objective is to observe and quantify the regular and irregular reflections and the diffractions of a blast wave on a real structure. In parallel, numerical simulations are performed with a home-made eulerian CFD code. The comparison with experimental results permits to discuss the capabilities and limitations of numerical blast predictions.
\end{abstract}

Keywords: Blast wave; Overpressure; Impulse; Reflection coefficient; Irregular reflection; CFD.

\section{NOMENCLATURE}

Parameters

$\mathrm{R} \quad$ distance to the charge

d diameter of the charge

E energy of the charge

$\mathrm{k}$ scale factor

$\mathrm{P} \quad$ pressure

$\Delta \mathrm{P} \quad$ overpressure

I impulse

$\mathrm{t}$ Phase duration

ta time of Arrival

$\mathrm{Cr}$ reflection coefficient

\section{Introduction}

Many industrial sites have the potential to cause major accidents because of the presence of a large amount of dangerous substances, such as oil products, natural gas, chemicals or explosives.Despite of considerable efforts towards safety many serious incidents have occurred these ten last years and renewed attention on such major hazards risks. Three recent explosions are taken as examples.Buncefield (United Kingdom) 2005 (HSE 2011, Atkinson and Cusco 2011), Jaipur (India) 2009(MoPNG 2011, Sharma et al. 2011) and
Greek letters

$\lambda \quad$ energetically scaled distance

$\gamma \quad$ heat capacity ratio

Superscripts

$+\quad$ positive

- negative

Subscripts

$0 \quad$ ambient

i Incident

r Reflected

Bayamon (Puerto Rico 2009 (CSB 2011) shared the characteristics of a violent blast generated by the ignition of a vapor cloud resulting from the spillage of a large amount of gasoline. In all cases, widespread structural damage to adjoining or off-site industrial units or commercial and residential buildings were observed. In these three different situations the collected evidences indicate that a deflagration to detonation transition is the most likely scenario.

If the main objective remains to prevent such events, focus is also put on mitigating the consequences 
and first and foremost limiting loss of life and injuries to occupants. In the case of buildings provided on a major hazard sites (e.g. chemical and petrochemical processing and storage plant) the ability to withstand the effects of blast loading is of utmost importance. Indeed, these buildings may not only serve to simply protect the plant and control systems from weather conditions, or only to be provided as accommodation for shelter. More importantly,they may be part of the overall containment strategy i.e. to prevent, control or mitigate major accident events.

In order to evaluate the most effective measures to protect occupants in a building, an analysis of the blast resistance of various structural components must be performed. A key factor of this evaluation is an accurate description of the blast loading. Simple approximate procedures based on published analytical or empirical relationships are usually used due to their intrinsic advantages such as speed and simplicity. The most often mentioned publications for simplified analysis are Baker et al. 1983, Kinney and Graham, 1985, and very extensively UFC-340-02, 2008(formerly TM5-1300). However, even when the building is assumed as a plain rectangular target, the interaction between the blast wave and the target remains a complex phenomenon:

- As the incident blast wave encounters a structure in its path, it is reflected on flat surfaces with amplified overpressures. The reflection is normal when the shock front is parallel to the plane of the surface and oblique when there is an incident angle. In this later case the reflection is classified as either regular or irregular when the reflectedwave overtakes the incident one and merges with it.

- As the blast wave is progressively travelling around the structure, it is also diffracted at corners in a complex manner, resulting in a decrease of the overpressures in these regions.

- Some elevations of the structure can experience a positive overpressure while others can simultaneously be subjected to a suction phase.

As a consequence, it is still not possible to analytically predict all the phenomena taking place during the interaction between the blast and a structure with a more complex profile such as a real building.

Advanced numerical methods such as computational fluid dynamics are required as an alternative to get a precise description of the blast loading for complex structures. The disadvantage is the computer resources required, which means that very few $3 \mathrm{D}$ simulations are accurately performed. There are several reasons for the limitation of quality of blast loading numerical estimations:

- Grid cell size: the impact of a mesh refinement was clearly established for example by Fairlie et al. 2000 and Catlin et al. 2001.

- Approximations in the representations of the initial blast environment: most of the calculations, avoiding the computationally expensive reactive-flow description for the combustion process, are based on a TNT equivalent method. But this method can give inaccurate results for vapor cloud explosions due to the disparity between the pressure and impulse compared with solid high explosives (Dusenberry 2010).

- The quality of used algorithms: discretization on Cartesian mesh, convergence rate, order of accuracy.

The main objective of this study is to evaluate the load magnitude and distribution on a real building when subjected to a global explosion. Well instrumented reduced-scale experiments are performed on a blast table where the blast wave is generated by the detonation of a gaseous mixture. These benchmark results are then used to assess the possibility offered by a single computer to numerically reproduce the main experimental observations.

\section{Experimentalprocess}

\subsection{Proposed reference case}

The building chosen is representative of an office located on an industrial site (Fig.1). It is 51 meters long, 18 meters large and 15.5 meters tall. This typical low-rise office building presents a complex profile due to the presence of an atrium with an apse and a hemispherical dome, a parapet surrounding the roof, and a plant room and stair towers at the rooftops.

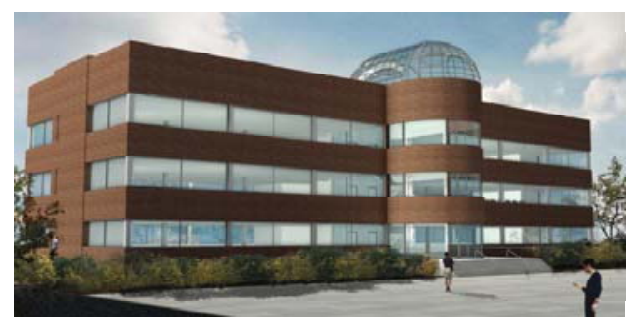

Fig. 1. Architect's impression of the building.

From the study of the consequences of a few typical industrial accidents involving detonation of condensed material (AZF, France 2001), detonation of a vapour cloud (Port Hudson, USA 1970), explosion of a vapour cloud (Buncefield, UK, 2005) or a BLEVE (Dagneux, France, 2007), it has been identified by INERIS (INERIS, 2014) that an incident overpressure between $140 \mathrm{hPa}$ and $200 \mathrm{hPa}$ could lead to significant structural damages on a structure.

\subsection{Reduced scale experiments}

Since blast experiments at real scale are prohibitively expensive and critical from a safety point a view, it is usual to perform small scale experiments in a secured experimental research laboratory. The experimental setup, presented in a previous work (Duong et al., 2012) consists of an instrumented blast table composed of four large plane panels $(132 \mathrm{~cm} \times 400 \mathrm{~cm})$. Shock waves are 
generated from the detonation of a semi-spherical stoichiometric mixture of propane and oxygen $(\mathrm{C} 3 \mathrm{H} 8+5 \mathrm{O} 2)$ confined in a soap bubble. The gaseous detonation, performed at ambient temperature and pressure conditions, is ignited by vaporizing a thin copper wire with a very high voltage $(7.5 \mathrm{kV})$. If similarity laws are satisfied, small scale results are transferable to the real scale.

The widely used Hopkinson-Cranz scaling law imposes the same scaled distance $\lambda=\frac{R}{\sqrt[3]{E}}$ for both real and reduced scale configuration.

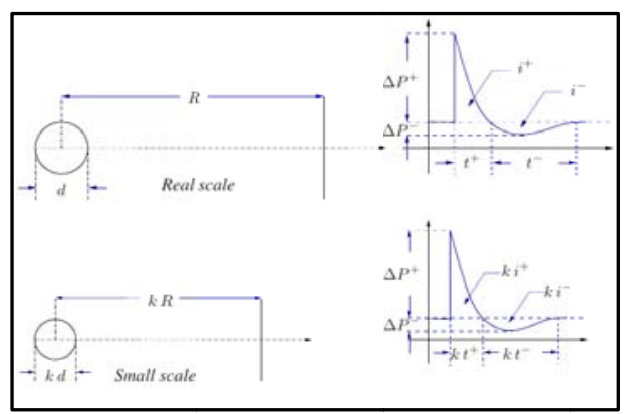

Fig. 2. Hopkinson-Cranz wave scaling.

This law states that an observer at a distance $k R$ from the source of the explosion will experience the same overpressure but an impulse and duration modified by the length scale factor $k$. This scaling law has been verified for aerial detonation by many experiments for a wide range of solid (Baker et al., 1983) or gaseous explosive charges (Brossard et al. 1985, Dorofeev 1996).

The choice of the scale factor results from a compromise between the size of the modular blast table and the expected incident overpressure range. $\Delta P^{+} \in[140,200] h P a$. The value used is $k=\frac{1}{60}$ which leads to a reduced model of $85.4 \times 31.4 \mathrm{~cm}^{2}$ with a height of $23 \mathrm{~cm}$. The specimen is made in poplar plywood panels except atrium in PVC pressure pipe with a wall thickness of $1 \mathrm{~cm}$ so that it can be considered as rigid (Fig.3).

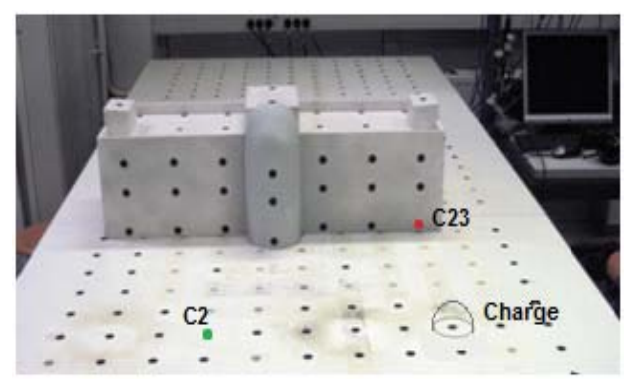

Fig. 3. Reduced scale experiment and charge position.

The bubble radius is set at $40 \mathrm{~mm}$ and the source of the gaseous explosion is located at $500 \mathrm{~mm}$ from the nearest point out of the reduced model. The expected reduced incident shock wave parameters are $\Delta \mathrm{P}^{+}=180$ mbar and $\mathrm{t}^{+}=0.26 \mathrm{~ms}$.

\subsection{Instrumentation}

The platen of each modular blast table is pierced by 99 holes covered with removable caps. Each hole can be used as a location either for the gaseous detonation initiation or to place pressure gauges.

Dynamic high frequency PCB quartz piezoelectric pressure sensors (113B26 and 113B28) associated with relevant signal conditioners are distributed either on the plane surface to study the incident shock wave propagation or/and on model to measure the reflected pressure. The pressure signals are recorded with very high speed NI digitizer (60MS/s); up to 24 signals can be simultaneously digitized and recorded.

\subsection{Proposed series of tests}

Three successive campaigns were performed so as:

- Todetermine the characteristics of the free field shock wave. This first campaign will be used to define blast curves that is to say polynomial interpolation of pressure, impulse and duration as a function of the reduced distance $\lambda\left(m M J^{-\frac{1}{3}}\right)$ :

$$
\log (X(\lambda))=a \log ^{3} \lambda+b \log ^{2} \lambda+c \log +d
$$

With $X=\Delta P^{+} / P_{0}$ or $\Delta I^{+} / \sqrt[3]{E}$ or $\Delta t^{+} / \sqrt[3]{E}$

- To determine the variation of reflection coefficients (ratio between the reflected overpressure and the incident overpressure: $C_{r}^{p}=\Delta P_{r}^{+} / \Delta P_{i}^{+}$) when the shock is impinging the front face of the specimen. In this second campaign three pressure sensors are installed on the table to ascertain the incident shock wave and the twenty one other sensors are positioned on the front of the model. To highlight and appreciate the transition from regular to irregular (Mach) reflection the model is rotated every 15 degrees around one of its corner, to keep the closest point at the fixed distance of $500 \mathrm{~mm}$.

- To determine the characteristics of the blast loading over the rest of the building - roof, sides and back - when the shock wave is travelling through the surface of the building. In this configuration the front face is not instrumented and once again, the model is rotated every 15 degrees around one of its corner.

\section{Numerical simulations}

As time goes on, most of the hydrocodes simulation tools such as AUTODYN, DYNA3D, EUROPLEXUS or MSC-DYTRAN can solve a wide variety of non-linear problems in solid, fluid and gas dynamics, and especially blast problem taking into account fluid structures interactions. However, for our blast problem, a simple Eulerian method is relevant since the blast loaded building is assumed to behave as a rigid body. The study of the loading produced by the blast wave can be divided in three main stages: 
- detonation phase where the explosive material ignites and the burn front expands in space;

- propagation phase where the blast waves propagates in the surrounding medium freely;

- interaction phase when the blast waves impinges the structure.

The explicit modeling of the detonation event is required in the near-field since the overpressure distribution is significantly modified by the shape of the charge and the real point of detonation in the charge. In the proposed experiments, the blast wave results from a hemispherical surface burst and the target is in far-field regarding the distance between the target and the point of detonation. As a consequence, the calculation of the fields variable remote from the source of detonation can be done by replacing the explosive by a hemispherical pressurized balloon. This approach is based on the initial work performed by (Brode, 1955). In this approach the boundary between the compressed air and the free-air can be seen as a fictitious membrane equivalent with the diaphragm in a shock-tube problem. The initial balloon parameters can be adjusted so that the pressure-time function resulting from the release of the compressed balloon can match the curve of an air blast wave (Catlin et al., 2001; Larcher, 2010).

The hemispherical surface-burst is equivalent to a spherical free-air burst so that before the blast wave interacts with an obstacle the problem presents a spherical symmetry. This spherical symmetry property justifies the study of a one-dimensional problem to be solved during the propagation phase. The output of the1D spherical solution is then remapped into the $3 \mathrm{D}$ domain with the building in it.

The numerical simulations conducted at Orleans University used a mesh generated with CARTFLOW (Deisteret al. 2003) and research solver METAS(Benselamaet al., 2009) developed at the University of Valenciennes. The resolution is based on the traditional upwind scheme and a two-stage explicit time integration technique, which gives an accuracy of the second-order in both space and time. In order to prevent numerical oscillations, which may occur in regions with strong gradients, the total variation diminishing minmod limiter was used.

As explained, the simulations were performed in two stages with the remapping of the results between each stage. A mesh refinement study was made, based on a criteria developed by (Catlin et al., 2001).

In this case the detonation was supposed to be located in a symmetry plane passing in the center of the building to halve the size of the computational mesh (Fig.4).

The mesh generated in $1 \mathrm{D}$ is fine enough $-0.1 \mathrm{~mm}$ to achieve convergence in both impulse and overpressure. The same refinement could not be achieved in 3D so that convergence is only ensured in impulse and the minimal mesh size near the model is $2.8 \mathrm{~mm}$, for a total of 10 million cells. The $3 \mathrm{D}$ simulation took about one month to run on a 2.7 $\mathrm{GHz}$ processor workstation with $128 \mathrm{~GB}$ of RAM.

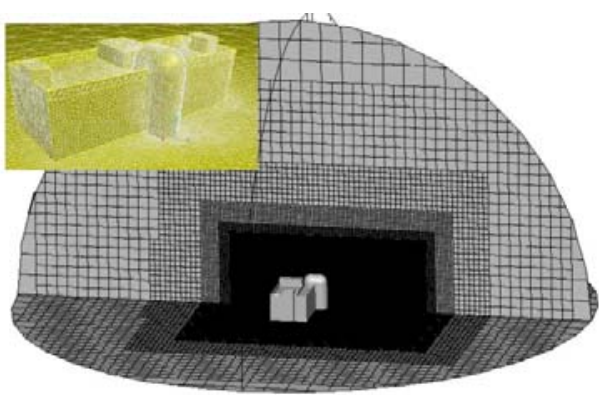

Fig. 4. Structure embedded in a cartesian mesh.

\section{Results and discussions}

\subsection{Repeatability and signal post processing}

In order to make sure that our experiments are solid and reliable, each configuration has been tested at least three times. Fig. 5 shows the pressure-time history and impulse-time curves obtained at sensor C23. This sensor is located directly in front of the center of detonation before any rotation of the model (Fig. 3 and Fig. 10). The impulse at current time $t_{c}$ is calculated form the pressure-time history as the integral of pressure from the time of shock arrival to the current time $t_{c}$. Fig. 5 reveals that the discrepancies between the different shots are less than $5 \%$.

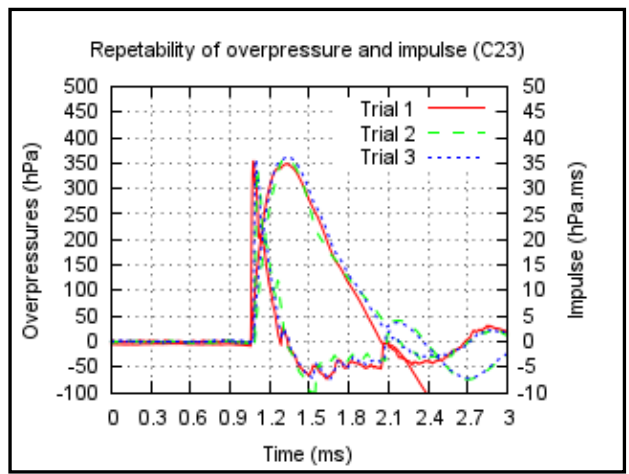

Fig. 5. Overpressure and impulse time histories showing the repeatability of the measures (reference sensor C23)

With the rotation of the model and the high number of sensors, more than 2000 pressures signals were to be analyzed.The more varying parameters are the overpressure $(4.15 \%)$, then the time of arrival $(1.64 \%)$, then the impulse $(0.96 \%)$.

However, for some experiments, high frequency noise was recorded (Fig.6). The comparison with a non-noisy record shows that the impulse value is not changed. Anyway, to avoid an inaccurate overpressure prediction, the decision was taken to 
filter the signals with a Butterworth filter with a cut-off frequency of $1 e^{5} \mathrm{~Hz}$.

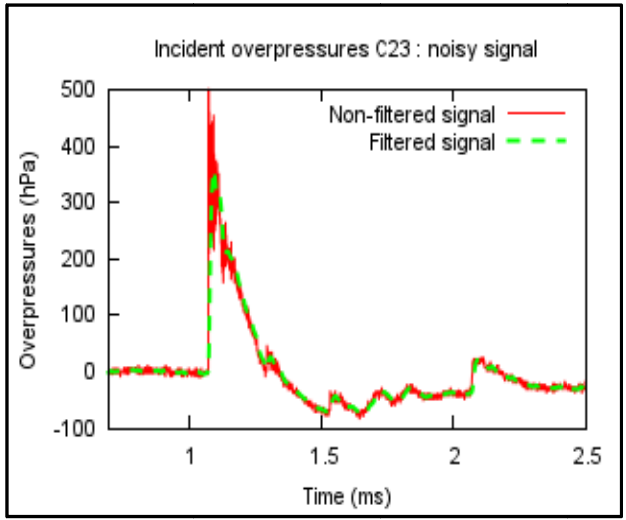

Fig. 6.Unfiltered and filtered signals

\subsection{Free Field overpressure}

The first campaign, performed without the specimen, allowed us to determine the variation of the blast wave characteristics with the reduced distance $\lambda$. The measurements confirm the polynomial interpolation law described in the part 2.4. The coefficients values, identified with a Levenberg-Marquardt least - squares curve fitting, are given in Table 1.

Table 1 Polynomial interpolation coefficient of reduced time of arrival, peak dimensionless overpressure, reduced impulse and reduced positive duration

\begin{tabular}{|c|c|c|c|c|}
\hline & $\frac{t_{a}}{\sqrt[3]{E}}$ & $\frac{\Delta P_{i}^{+}}{P_{0}}$ & $\frac{I_{i}^{+}}{\sqrt[3]{E}}$ & $\frac{t_{i}^{+}}{\sqrt[3]{E}}$ \\
\hline$\lambda$ & $0.7 \div 8.2$ & $0.7 \div 8.2$ & $0.7 \div 8.2$ & $0.7 \div 8.2$ \\
\hline$a$ & 0 & 0 & -0.102 & 0 \\
\hline$b$ & -0.126 & 0.125 & 0.118 & -0.161 \\
\hline$c$ & 1.57 & -1.703 & -0.754 & 0.675 \\
\hline$d$ & 0.259 & 0.085 & -1.207 & -0.053 \\
\hline
\end{tabular}

\subsection{Influence of the incidence angle on overpressure}

In most of real accidents, the blast wave will strike the building surface at oblique incidence. As explained in part 2.4, in our experiments the model is rotated around one of its corner between $0^{\circ}$ and $90^{\circ}$ while the center of detonation remains located on the side of the model, as showed on Fig.3. As a consequence the distance between the source and the pressure sensors is not always the same except for sensors in the right corner (Fig. 3). Accordingly, we first study the variation of overpressures recorded by sensors C23 during the rotation (Fig.7). A general decrease of the overpressures is observed, except near a rotation angle of $60^{\circ}$. This phenomenon is regularly observed and described in the literature as irregular or Mach reflection. The Mach reflection is an irregular reflection occurring when the reflected shock wave catchs up with the incident one.

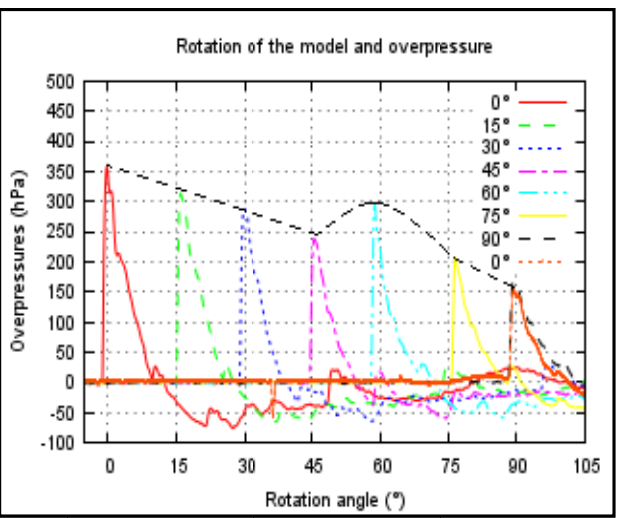

Fig. 7. Influence of the rotation angle.

This critical angle value, depending on the incident overpressure, can be numerically computed as the value at which the resolution of the regular reflexion problem becomes impossible (Ben-Dor, 2007). There is a good agreement since for an incident overpressure $\Delta P^{+}=170 \mathrm{hPa}$, the computed critical value is $57^{\circ}$. It can also be seen that the $90^{\circ}$ reflected overpressure, which in facts becomes a side on overpressure, is as expected equivalent to the incident one.

\subsection{Reflection coefficient on sensor C23}

Knowing the incident blast wave, the blast loading on a structureis often defined using reflection coefficients assuming a similarity between the positive phase durations between incident and reflected blast waves (Baker et al. 1985). The pressure sensor $\mathrm{C} 22$ is one of the three reference free-field sensors located at the same distance from the source as sensor C23. Thus, we can compare the actual incident and reflected signals on the same test. Fig. 8 shows this comparison. For a normal reflection, an analytical expression based on Rankine-Hugoiot equations is available:

$C_{r}^{p}=\frac{\Delta P_{r}}{\Delta P_{i}}=2+\frac{\Delta P_{i}(\gamma+1)}{(\gamma-1) \Delta P_{i}+2 \gamma P_{0}}$

For an incident peak overpressure $\Delta P_{i}^{+}=170 \mathrm{hPa}$, the theoretical value is 2.14. The experimental reflected peak overpressure value is $\Delta P_{r}^{+}=$ $360 \mathrm{hPa}$ which provides a value of 2.12 in very good agreement.

It is interesting to notice that when one compares the reflected overpressure with the incident overpressure multiplied by the reflection coefficient, the curves are superimposed on each other during the positive phase as seen in Fig. 9,this positive phase being the only one taking into account in most of structural blast analysis (UFC-340-02, 2008). 


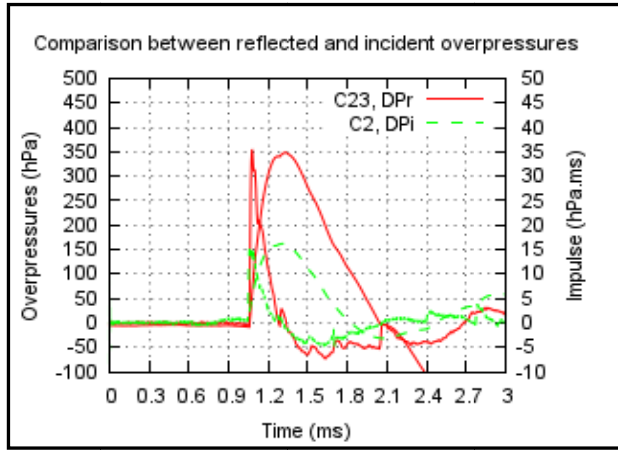

Fig. 8. Comparison between incident and reflected shock wave.

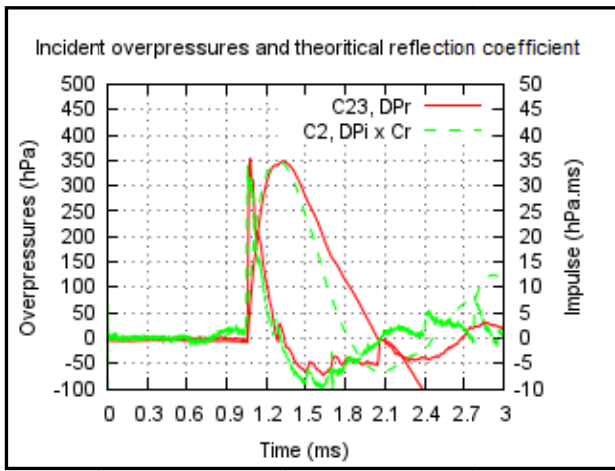

Fig. 9. Experimental results: comparison between $\Delta \mathrm{P}_{\mathrm{i}}^{+} \times \mathrm{C}_{\mathrm{r}}$ and $\Delta \mathrm{P}_{\mathrm{r}}^{+}$.

The same comparison is performed with the simulated experiment. The numerical reflection coefficient is equal to the experimental one (2.12).

\subsection{Pressure variation on the whole building facadewith variable incidence angle}

Since the whole building's façade was instumented with pressure sensors, the variation of overpressures along the building height can be evaluated. A particular attention can also be devoted to the effect of the atrium. Depending on the angle of incidence this one may indeed create shadow areas but also areas of multiple reflections in the corner. Fig. 10 shows the position of the sensors in front of the model.

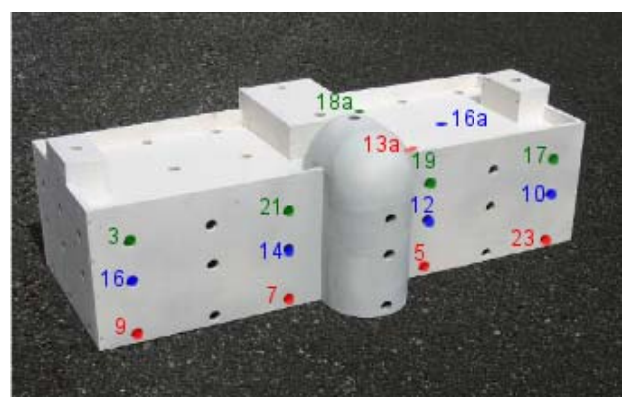

Fig. 10. Position of the sensors in front on the model.
For all of these sensors, the polynomial law established during the free field experiment (Part 4.3) was used to evaluate the incident overpressures, hence the reflection coefficient. The reflection coefficients computed from the numerical simulations presented in Part III are also carried forward. The incident blast wave overpressures are deduced from the 1D free-field simulations. The next figures (Fig. 11, Fig 12. and Fig 13.) present the change in reflection coefficients according to the height at different places of the building's facade.

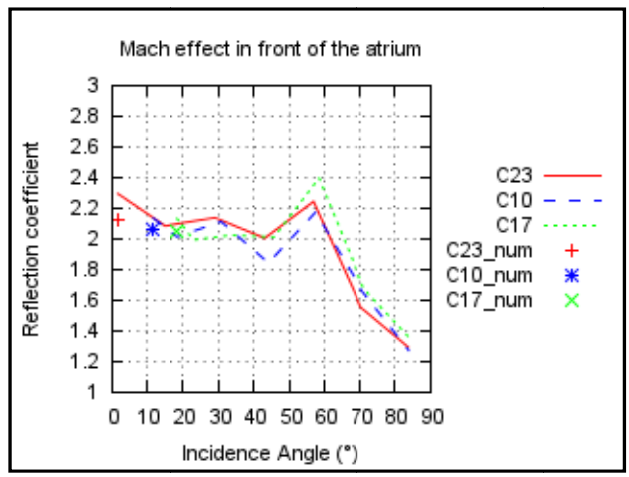

Fig. 11. Reflection coefficient, right corner facing explosion.

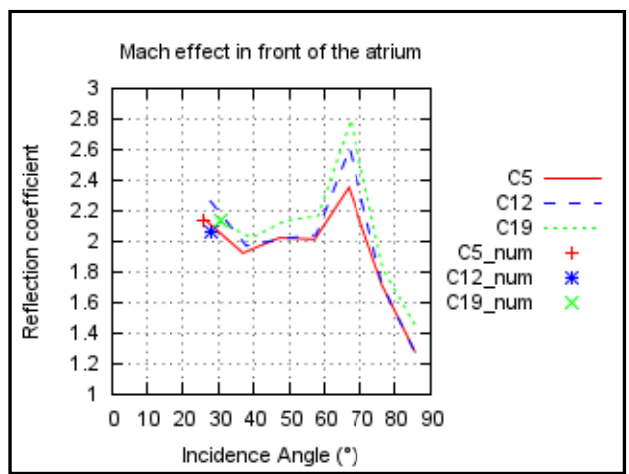

Fig. 12. Reflection coefficient, in front of the atrium (lighted area)

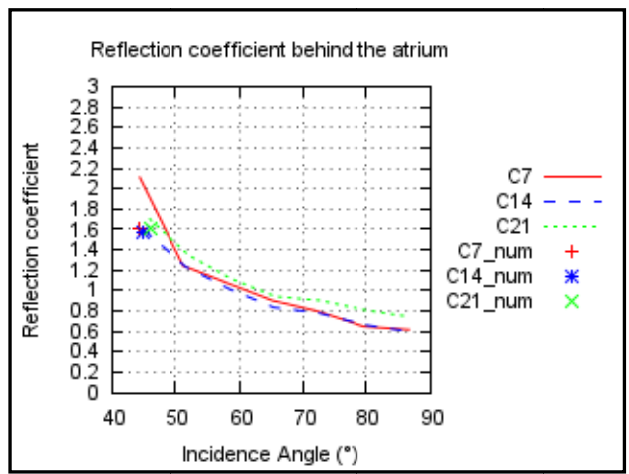

Fig. 13. Reflection coefficient, closest column behind the atrium (shadowed area) 


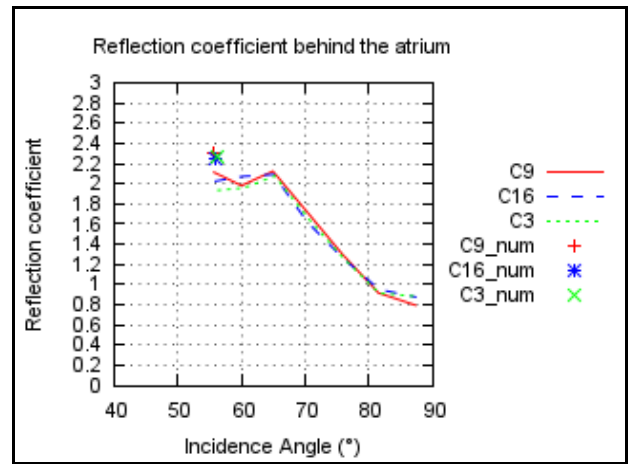

Fig. 14. Reflection coefficient, furthest column behind the atrium

For all the vertically aligned sensors located in the lighted area, we can notice an increase of the reflection coefficient between $50^{\circ}$ and $60^{\circ}$ (Fig.11 and Fig. 12). As already mentioned, this sharp variation can be associated with the Mach reflection (Fig. 7). It must be emphasized that this effect for low incident overpressure has been open to debate and is still considered as negligible (TNO, 2005). Caution should be taken when interpreting the results of reflected overpressures for the sensors the closest to the atrium (Fig. 12). The peak reflected overpressure - almost three times the incident oneis interpreted as the combination of two concomitant effects: Mach reflection and recombination of two reflected shock waves (on the atrium and on the building). Additional tests need to be performed to assess the validity of this analysis.

On the contrary, Fig. 13 and Fig. 14 show an important decrease of the reflection coefficient behind the atrium.

This is explained by the rotation of the model, which put sensors in the shadowed area behind the atrium. A limit value close to 0.6 can be observed for the column the closest to the atrium $(\mathrm{C} 7, \mathrm{C} 14$, and $\mathrm{C} 21$ ).

As the vertically aligned sensors are at a greatest distance from the atrium, the limit value approaches 1 (Fig. 14) as if the effect of the diffraction of the shockwave tends to vanish.

The reflection coefficients on the rear wall (when the model is not rotated) or the side walls (when the model is rotated 90 degrees) are not different with a mean value close to 0.5 . Otherwise, the curves are similar to the ones presented in Fig. 11 and Fig. 12.

Reflection coefficients on the roof show different values (Fig.15). Most of the time, they have a value within the range 0.4 to 0.6 . This means that the overpressure is quite different from the side on value. This discrepancy is explained by:

- the fact that in our experiments the shockwave is spherical and not supposed to be planar;

- the presence of a parapet wich generates ad iffraction of the shockwave.

The three sharp increases in reflection coefficients observed in Fig. 15 for different angular for sensors $13 \mathrm{a}$ and $18 \mathrm{a}$ are explained by reflections of the

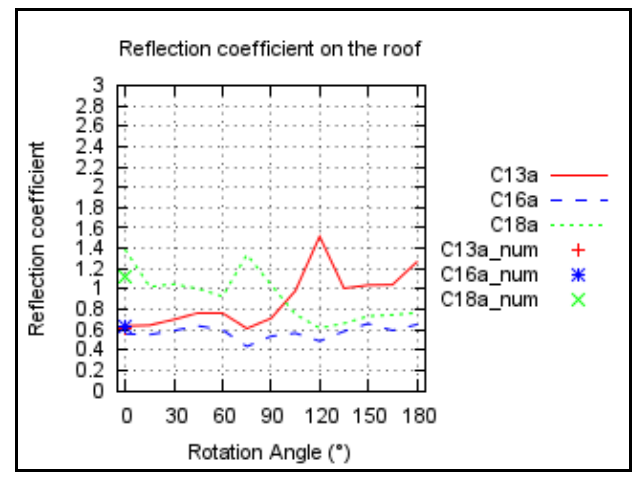

Fig. 15. Reflection coefficient on the roof

diffracted shockwave on parapet, atrium and plant room. Globally, it can be considered that the parapet plays the role of a protective barrier.

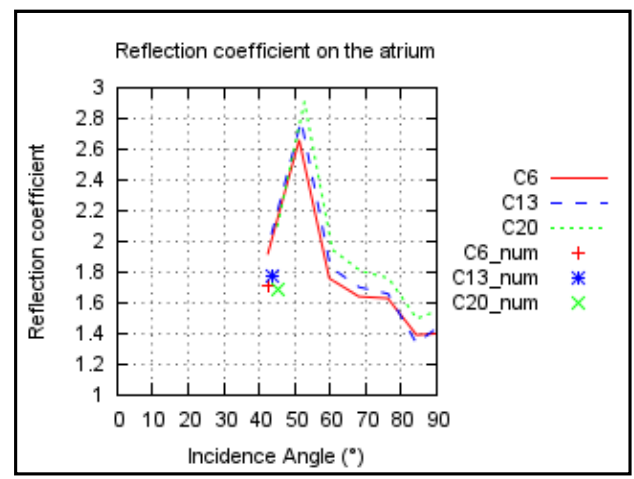

Fig. 16. Reflection coefficient on the atrium

The comparison of the numerical and experimental reflection coefficients (Fig.11 to 15.) shows a good agreement in all situations. This trend should be confirmed by ongoing numerical simulations with a rotated model in order to numerically quantify the Mach reflection.

This good agreement is confirmed by a comparison of pressure-time histories of sensors located all around the building. The discrepencies between numerical and experimental data are less than $10 \%$ in term of overpressure and less than $5 \%$ in term of time of arrival.

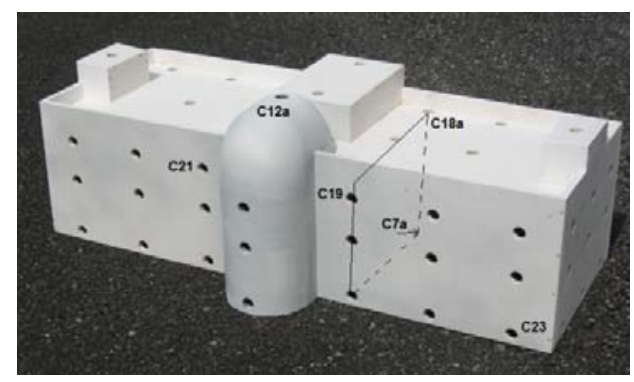

Fig. 17. Position of the sensor compared with the numerical results 


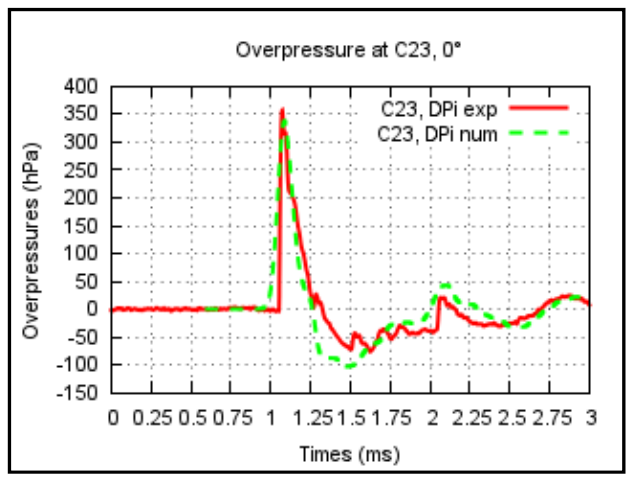

Fig. 18. Comparison for the reference sensor

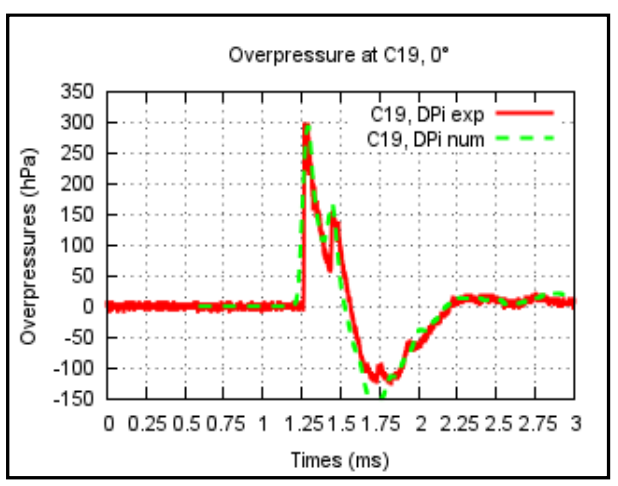

Fig. 19. Comparison in front of the atrium

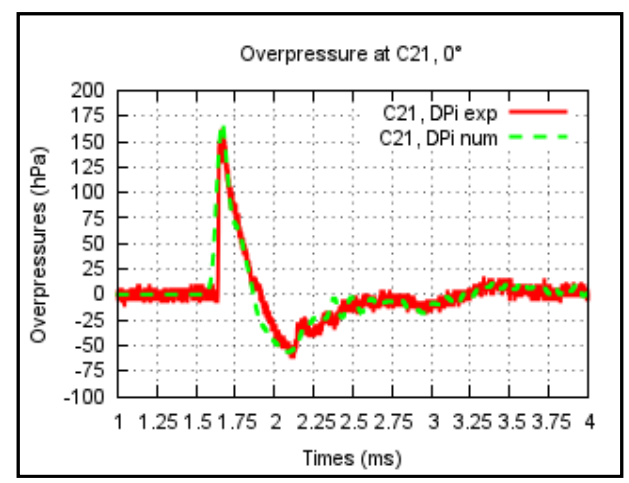

Fig. 20. Comparison behind the atrium

Because of the mesh refinement some multiples reflections may be overlooked numerically but the reflected coefficient is good thanks to the conservative impulse (Fig. 23).

\section{CONCLUSION}

In this paper, we experimentally and numerically studied the reflection of a shockwave on a complex building. We ascertain the possibility of an eulerian code to correctly predict the parameters of an overpressure located in the shadowed area. Especially, the time related parameters matches the experimental results thank to the use of a compressed balloon method using perfect gas

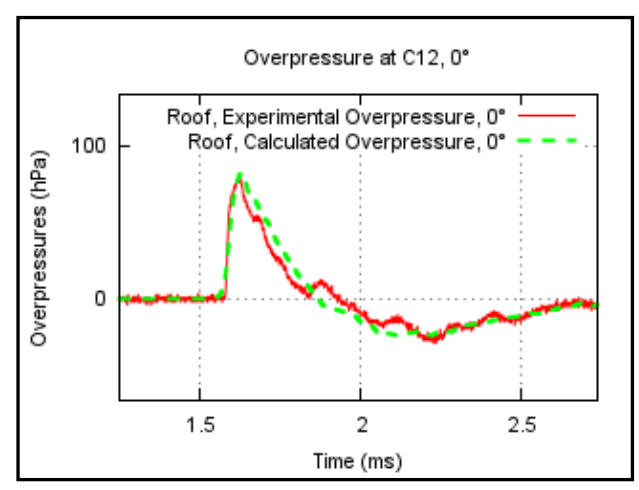

Fig. 21. Comparison on the roof

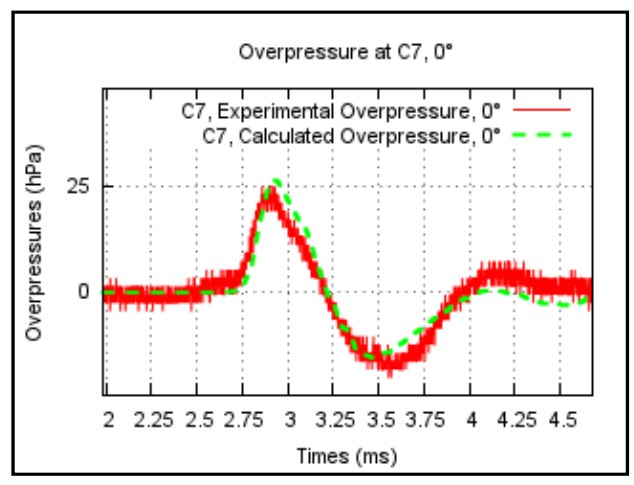

Fig. 22. Comparison on the back

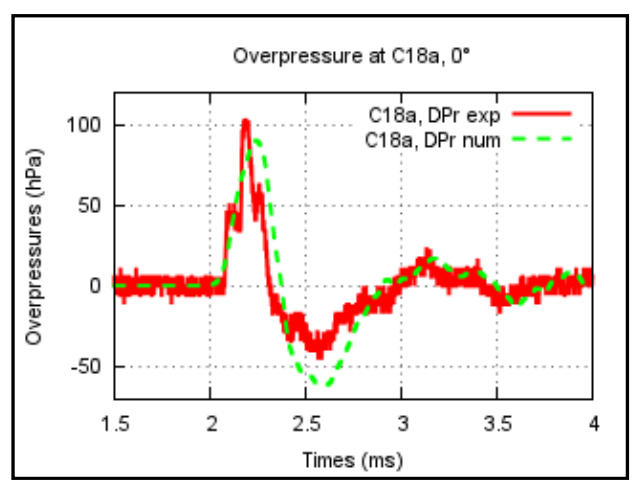

Fig. 23. Comparison in a corner

equation of state. These results can be applied to similar situation, for any materials, and gives a good alternative to the use of TNT equivalent and JWL equation of state.

Mach reflection of blast waves with low overpressures should be investigated.

\section{ACKNOWLEDGEMENTS}

The research leading to these results has received funding from the European Union's Research Fund for Coal and Steel (RCFS) under grant agreement no. RFSR-CT-2013-00020 
L. Blanc et al. /JAFM, Vol. 9, Special Issue 1, pp. 121-129, 2016.

\section{REFERENCES}

Atkinson G. and L. Cusco (2011).Buncefield.A violent, episodic vapour cloud explosion, Process Saf. Environ. Prot., 89, pp. 360-370.

Baker, W. E., P. A Cox., P. S .Westine., J. J Kulesz, and R. A Strehlow, (1983). Explosion hazards and evaluation, Elsevier, Amsterdam.

Ben-Dor, G. (2007).Shock Wave Reflection Phenomena, $2^{\text {nd }}$ edition, Springer, Dordrecht.

Benselama, A. M., M William-Louis.,andF Monnoyer.(2009).A 1D-3D mixed method for the numerical simulation of blast waves in confined geometries, Journal of Computational Physics, $228 \mathrm{n}^{\circ} 18$, pp. 6796-6810.

Brode, H. L. (1955). Numerical solutions of spherical blast waves, Journal of Applied Physics, No. 6, vol. 26, p. 766-775.

Brossard J, J. C. Leyer, D.Desbordes, JR Saint Clouds HendrickxS,JL Garnier, A Lannoy, and J Perrot (1985). Air blast unconfined gaseous detonations, Dynamics of Shock Waves Explosisions and Detonations, Vol.94, Progress in Astronautics and Aeronautics, vol.94.

Catlin C., M. Ivings and S. Myatt. (2001). Explosion Hazard Assessment: A Study of the Feasability and Benefits of Extending Current HSE Methodology to take Account of Blast Sheltering, Report HSL/2001/04.

Dorofeev, S. B. V. P. Sidorov, M. S Kuznetsov, A. E. Dvoinishnikov, V. I Alekseev and Efimen, ko A. A. (1996). Air blast and heat radiation from fuel-rich mixture detonations, Shock Waves 6:21-28 9.

Deister, F. E. Waymel., F. Hirschel and F. Monnoyer (2003). Self-organizing hybrid Cartesian grid generation and application to external and internal flows, Numerical Flow Simulation III, Notes on Numerical Fluid Mechanics and Multidisciplinary Design, Vol. 82), pp. 18-29.

Duong, D. H., J. L Hanus, L Bouazaoui, O Pennetier, J Moriceau, and G Prod'homme,., Reimeringer, M.(2012).Response of a tank under blast loading - Part I: Experimental characterization, European Journal of Environmental and Civil Engineering 16 (9), p. 1023-1041.
Dusenberry, D. O. (2010).Handbook for blast-resistant design of buildings, Hoboken, N.J., J. Wiley.

Fairlie, G. E. N. F. Johnson and K. C. Moran. (2000). Validated numerical simulations of blast loads on structures. In: 16th International Symposium on Military Aspects of Blast andShock.

INERIS (2014). Action on buildings due to explosions: Pressure-time history due to realindustrial explosions, research report, DRA-13-132890-14078C.

Kinney, G. F. and K. J. Graham. (1985). Explosive shocks in air, $2^{\text {nd }}$ edition, Berlin and New York, Springer-Verlag, $282 \mathrm{p}$.

Larcher, M. and F. Casadei. (2010). Explosions in complex geometries - a comparison of several approaches, International Journal of Protective Structures, 1 (2), pp. 169-195.

CPR16E (2005). Committee the Prevention of Disaster (CPR). The Green Methods for the determination of possible damage, Part 2B: Effects of explosion on strcutrures.

UFC-340-02 (2008).Unified Facilities Criteria, Structures to resist the effects of accidental explosions, Washington DC: US Department of Defense.

Sharma, R. K., B. R.Gurjar., S. R.Wate., S.P.Ghuge. and R Agrawal.(2013).Assessment of an accidental vapour cloud explosion: Lessons from the Indian Oil Corporation Ltd., accident at Jaipur, India, J. Loss Prevention Process, Ind. 26, p. 82-90.

Health and Safety Executive (HSE) (2011).TheBuncefield Incident 11 December 2005, Major IncidentInvestigation Board Final Report, Vol 1 and 2, UK.

US Chemical Safety and Hazard Investigation Board (CSB), (2015). Caribbean Petroleum Tank Terminal Explosion and Multiple Tank Fire, Final Investigation Report, USA.

Ministry of Petrolium and Natural Gas (MoPNG) (2011).Independent Inquiry Committee Report on Indian Oil Terminal Fire at Jaipu, Fire Accident Investigation Report, Oil Industry Safety Directorate, Government of India. 